\title{
Therapeutic nanoplatforms and delivery strategies for neurological disorders
}

\author{
You Jung Kang, Eric Gerard Cutler and Hansang Cho* ${ }^{*}$
}

\begin{abstract}
The major neurological disorders found in a central nervous system (CNS), such as brain tumors, Alzheimer's diseases, Parkinson's diseases, and Huntington's disease, have led to devastating outcomes on the human public health. Of these disorders, early diagnostics remains poor, and no treatment has been successfully discovered; therefore, they become the most life-threatening medical burdens worldwide compared to other major diseases. The major obstacles for the drug discovery are the presence of a restrictive blood-brain barrier (BBB), limiting drug entry into brains and undesired neuroimmune activities caused by untargeted drugs, leading to irreversible neuronal damages. Recent advances in nanotechnology have contributed to the development of novel nanoplatforms and effective delivering strategies to improve the CNS disorder treatment while less disturbing brain systems. The nanoscale drug carriers, including liposomes, dendrimers, viral capsids, polymeric nanoparticles, silicon nanoparticles, and magnetic/metallic nanoparticles, enable the effective drug delivery penetrating across the BBB, the aforementioned challenges in the CNS. Moreover, drugs encapsulated by the nanocarriers can reach further deeper into targeting regions while preventing the degradation. In this review, we classify novel disease hallmarks incorporated with emerging nanoplatforms, describe promising approaches for improving drug delivery to the disordered CNS, and discuss their implications for clinical practice.
\end{abstract}

Keywords: Nanomedicine, Nanoparticle, Drug delivery, Targeted delivery, Neurological diseases, Central nervous system (CNS), Blood-brain barrier (BBB), Biomarker, Glioblastoma (GBM), Alzheimer's diseases (AD), Parkinson's diseases (PD)

\section{Introduction}

It has been challenge to improve prognose of diseases, such as brain tumor, Alzheimer's diseases (AD), and Parkinson's diseases (PD) [1-3] in a central nervous system (CNS) compared to other body parts. An enormous effort has been made for discovery and development of CNS drugs that can elude toward new and effective treatments; however, more than $90 \%$ of newly proposed drugs have failed to receive clinical approvals from the US Food and Drug Administration (FDA) [4]. One of major difficulties for the development of CNS treatments is the presence of a tighten blood-brain barrier (BBB) limiting

\footnotetext{
*Correspondence: h.cho@uncc.edu

Department of Mechanical Engineering and Engineering Science, Center for Biomedical Engineering and Science, Department of Biological

Sciences, The Nanoscale Science Program, University of North Carolina at Charlotte, Charlotte, NC, USA
}

drug entry to the CNS region [5]. Additionally, non-targeted delivery of diagnostic reagents or therapeutic drugs can cause detrimental effects on the neuronal cells and glial cells, extremely sensitive cellular components in the CNS maintaining brain functions and homeostasis. In this regard, it is an urgent issue to design and develop novel delivery platforms bearing the diagnostic reagents or therapeutic drugs for the neurological disorders.

Nowadays, nanomedicines in various medical fields hold a great promise to deliver the imaging agents or treatments for CNS diseases due to their beneficial features. The size of nanomedicines, operating in the range of 10-200 nm, achieve not only increased physiological properties of reactivity, strength, surface area, sensitivity, and stability [6], but also enhanced penetration properties across a BBB and deep into diseased brain tissues [7]. The drug encapsulation can prevent the degradation of drugs in the process of delivery, and the controlled 
release offers a safely delivery system by achieving the delivery of cytotoxic drugs to the disease area with appropriate dosage without harming healthy tissue. The modification on the surface of nanomedicines further enhance the $\mathrm{BBB}$ penetration as well as disease-targeting efficiency. Recently developed versatile nanomedicines can serve both diagnostic and multimodal treatment functions at the same time and proven their excellent potentials for targeting CNS diseases; a number of optimizations are still required for the future clinical purpose.

In this review, we discuss the emergence of nanomedicines for treating CNS diseases and their recent progress as well as future potentials. This paper includes the brief description of neurological diseases with signature markers widely employed in the nanomedicine area, the list of currently developed nanomedicines applied for drug delivery and sensing purposes, and promising strategies to improve $\mathrm{BBB}$ penetration and/or disease-targeting efficiency of nanomedicines. At the end, we briefly discuss the potentials of nanomedicines and future directions to achieve success in the clinical trials.

\section{List of CNS diseases and diagnosis/treating strategies}

\subsection{Glioblastoma (GBM)}

GBM is the most common and deadliest form of the primary brain tumor since the median survival rate is 14 months, even following aggressive multimodal therapy, such as surgery, radiation, chemotherapy, and their combined treatments [1]. This indicates the urgent need for the development of an effective strategy to eliminate brain tumors. Through extensive studies on the discovery of novel markers highly expressed on the brain tumors, their application in several nanomedicines functionalized with targeting ligands significantly succeed on improving the elimination of brain tumors [8-17]. Recently, immunotherapy targeting immune checkpoint receptors expressed on adaptive immune cells enhancing immune surveillance has emerged as another anticancer approach and led to explosion of clinical trials [18]. The biomarkers targeting the brain tumors or immune cells are summarized in Table 1. However, the intratumor heterogeneity varying between individual patients reduces the targeting efficiency and fails to eliminate the tumors completely

Table 1 List of CNS diseases and current strategies targeting the disease hallmarks

\begin{tabular}{|c|c|c|c|c|c|}
\hline & Biomarker & Targeting strategy & Purpose & Examples & Refs. \\
\hline \multirow[t]{8}{*}{ GBM } & MGMT methylation & Methylation-primer & Diagnosis & PCR-mMGMT & {$[149]$} \\
\hline & PDGFRA mutation & PDGFRS-primer & Diagnosis & qPCR, qPCR-SSCP & {$[150]$} \\
\hline & & PDGR inhibitor & GBM treatment & $\begin{array}{l}\text { Sorafenib } \\
\text { Matinib } \\
\text { Tandutinib } \\
\text { Dasatinib }\end{array}$ & {$[151]$} \\
\hline & IDH mutation & Anti-R132H-IDH1 & Diagnosis & Immunohistochemistry & {$[152]$} \\
\hline & EGFR mutation & Anti-EGFRvIII & Diagnosis & Immunohistochemistry & {$[153]$} \\
\hline & & EGFR inhibitor & GBM treatment & $\begin{array}{l}\text { Erlotinib } \\
\text { Nimotuzumab } \\
\text { Gefitinib } \\
\text { Cetuximab }\end{array}$ & {$[151]$} \\
\hline & PD-1 & PD-1 inhibitor & GBM treatment & $\begin{array}{l}\text { Nivolumab } \\
\text { Pembrolizumab }\end{array}$ & {$[18]$} \\
\hline & CTLA-4 & CTLA-4 inhibitor & GBM treatment & Ipilimumab & {$[18]$} \\
\hline \multirow[t]{4}{*}{$A D$} & $A \beta$ oligomer & $A \beta$ aggregation inhibitor & AD treatment & Tramiprosate & {$[21]$} \\
\hline & A $\beta$ plaque & $A \beta$ aggregation inhibitor & AD treatment & Colostrinin & {$[22]$} \\
\hline & & A $\beta$ PET ligand & PET imaging & $\begin{array}{l}\text { CRANAD } \\
\text { NIAD }\end{array}$ & {$[154]$} \\
\hline & Tau & Tau aggregation inhibitor & AD treatment & $\begin{array}{l}\text { Astemizole } \\
\text { Lansoprazole }\end{array}$ & {$[23]$} \\
\hline \multirow[t]{5}{*}{ PD } & DAT & Dopamine & PD treatment & L-DOPA & {$[27]$} \\
\hline & & Dopamine agonist & PD treatment & Bromocriptine & {$[28]$} \\
\hline & & D2/D3 radioligands & $\begin{array}{l}\text { SPEC imaging } \\
\text { PET imaging } \\
\text { PET imaging }\end{array}$ & $\begin{array}{l}{ }^{123} \text { /-iodolisuride } \\
{ }^{11} \mathrm{C} \text {-Raclopride } \\
{ }^{18} \text { F-Fallypride }\end{array}$ & {$[155]$} \\
\hline & & Cocaine derivatives & $\begin{array}{l}\text { PET imaging } \\
\text { PET imaging }\end{array}$ & $\begin{array}{l}{ }^{125} \mathrm{I}-\mathrm{RTI}-55 \\
{ }^{76} \mathrm{Br}-\mathrm{PE} 2 \mathrm{Br}\end{array}$ & [155] \\
\hline & a-Synuclein & Anti-a-synuclein & Diagnosis & ELISA & [143] \\
\hline
\end{tabular}


causing the recurrence. Thus, the development of promising nanomedicines equipped with the innovative targeting and the multimodal treatment systems is now highly required for removal of cancers completely.

\subsection{Alzheimer's disease (AD)}

$\mathrm{AD}$ is a progressive and neurodegenerative brain disorder, which is the most common cause of dementia resulting in loss of memory, thinking and language skills, and behavioral changes [19]. However, no definitive cure for $\mathrm{AD}$ exists due to lack of knowledge of its molecular and intercellular mechanisms. Key signatures of $\mathrm{AD}$ progression include deposition of amyloid-beta $(A \beta)$ peptides, neurofibrillary tangle formation of phosphoric tau proteins, and detrimental neuroinflammation in brains leading to synaptic impairment and neuronal loss [20]. The $A \beta$ plaques around $A D$ brains, soluble $A \beta$ in the cerebrospinal fluids (CSFs), tau proteins presenting in the CSF are major biochemical markers, which have been targeted for the diagnosis purposes. Current therapeutic approaches are focusing on the inhibition of $A \beta$ plaque/ tau tangle formation and neutralization of their aggregations around neurons (Table 1) [21-23]. Other clinically approved drugs can only alleviate symptoms and delay AD progression by providing neurotransmitters, which promote interactions between neurons in AD brains [24]. In this regard, the discovery of novel AD markers and development of nanomedicines targeting the $\mathrm{AD}$ markers have been on-demand.

\subsection{Parkinson's disease (PD)}

$\mathrm{PD}$ is the second common neurodegenerative disease occurring primarily in the substantia nigra causing development of bradykinesia and tremors of cardinal motor functions [3]. The major hallmark found in PD models is the decreased level of dopamine transporters (DATs), which play curial roles in the uptake of dopamine by dopaminergic neurons and proceed the communications between neurons; therefore, the reduced dopamine delivery is contributed to the significant loss of neuronal functions [25]. Another marker is the accumulation of $\alpha$-synuclein in the Lewy bodies, but their underlying mechanisms leading to PD dementia are not clearly defined yet [26]. These two markers have employed for PD diagnosis and treatment and are summarized in Table 1. Unfortunately, there is no current cure for the PD condition, but the treatment of initial PD stages using levodopa (L-DOPA), the precursor of dopamine, or L-DOPA agonist is the typical way to retard the progression of PD [27, 28]. However, untargeted delivery of L-DOPA can attack the peripheral system causing the adverse cardiovascular effect and dyskinesia [29]. Therefore, the neurotransmitters for the PD treatment should be encapsulated in the proper delivery system that allows the penetration of $\mathrm{BBB}$, not other peripheral vessels.

\section{Classification of nanomedicine platforms}

3.1 Lipid-based nanoparticles: liposomes and exosomes

Nanoliposomes, the nanosized and spherical forms of enclosed lipid bilayers, have extensively applied as a nanocarrier of therapeutic drugs, imaging agents, and genes for treatment and/or diagnosis of CNS diseases [8-11, 30, 31]. The liposomes are composing of a hydrophilic core and one or more hydrophobic spaces surrounded by lipid bilayers, and this unique amphiphilic structure enables encapsulation of both hydrophobic and hydrophilic compounds. Hydrophilic therapeutic drugs and DNAs have been loaded in the core space of nanoliposomes, which have been applied for the delivery of these compounds to CNS diseases area [11]. Moreover, various hydrophobic compounds have been entrapped into the hydrophobic interface between the lipid bilayers and applied in therapeutic as well as diagnostic purposes [10]. The common method for the nanoliposome preparation is the lipid film rehydration method, followed by either freezing-thawing cycles or sonication [32]. To yield an unilamellar liposomal suspension with a low polydispersity, this method has been combined with the extrusion technique equipping a porous membrane (pore size $<0.2 \mu \mathrm{m}$ ), which defines the size of nanoliposomes [33]. To further precisely control the size of nanoliposomes, several sophisticated techniques, such as microfluidic-based platforms, has been combined with the traditional nanoliposome preparation methods [34]. The major advantages of nanoliposomes are their high biocompatibility, excellent flexibility to tune the biophysical and physiochemical properties, and well-established preparation methods [35]. In order to achieve improved blood circulation and targeting efficiency, the surface of nanoliposomes has been further decorated with polymers (Polyethylene glycol (PEG), etc.) (Fig. 1a) [36], polysaccharides (dextran [37], mannose [38, 39], etc.), targeting molecules (transferrin [10], T7 peptide [11], Interleukin$[9,13]$ Arginyl-glycyl-aspartic acid (RGD)-based peptides [10], etc.), or aptamers (GBM128 [40], etc.). The list of nanoliposomes for targeting CNS diseases are summarized in Table 2. Despite the described improvements, circulation life-time of nanoliposomes in the blood is still short, and the leakage of compounds through the lipid bilayers and the low stability toward mechanical shear stress as well as osmotic pressure have remained as major obstacles for the clinical uses [41].

Exosomes are recently proposed nanoliposomes derived from the cellular endosomes, and they can encapsulate drugs and target CNS diseases upon the harvesting exosomes followed by post modifications 

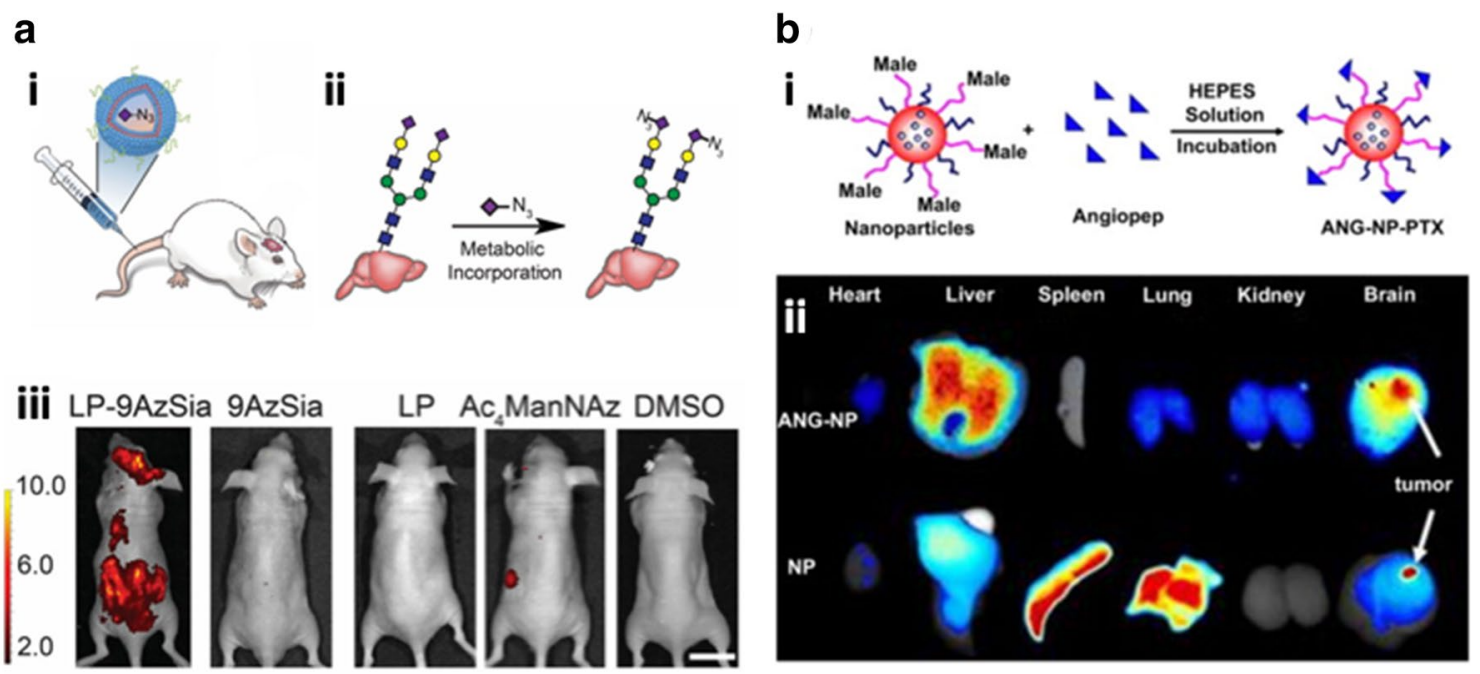

d
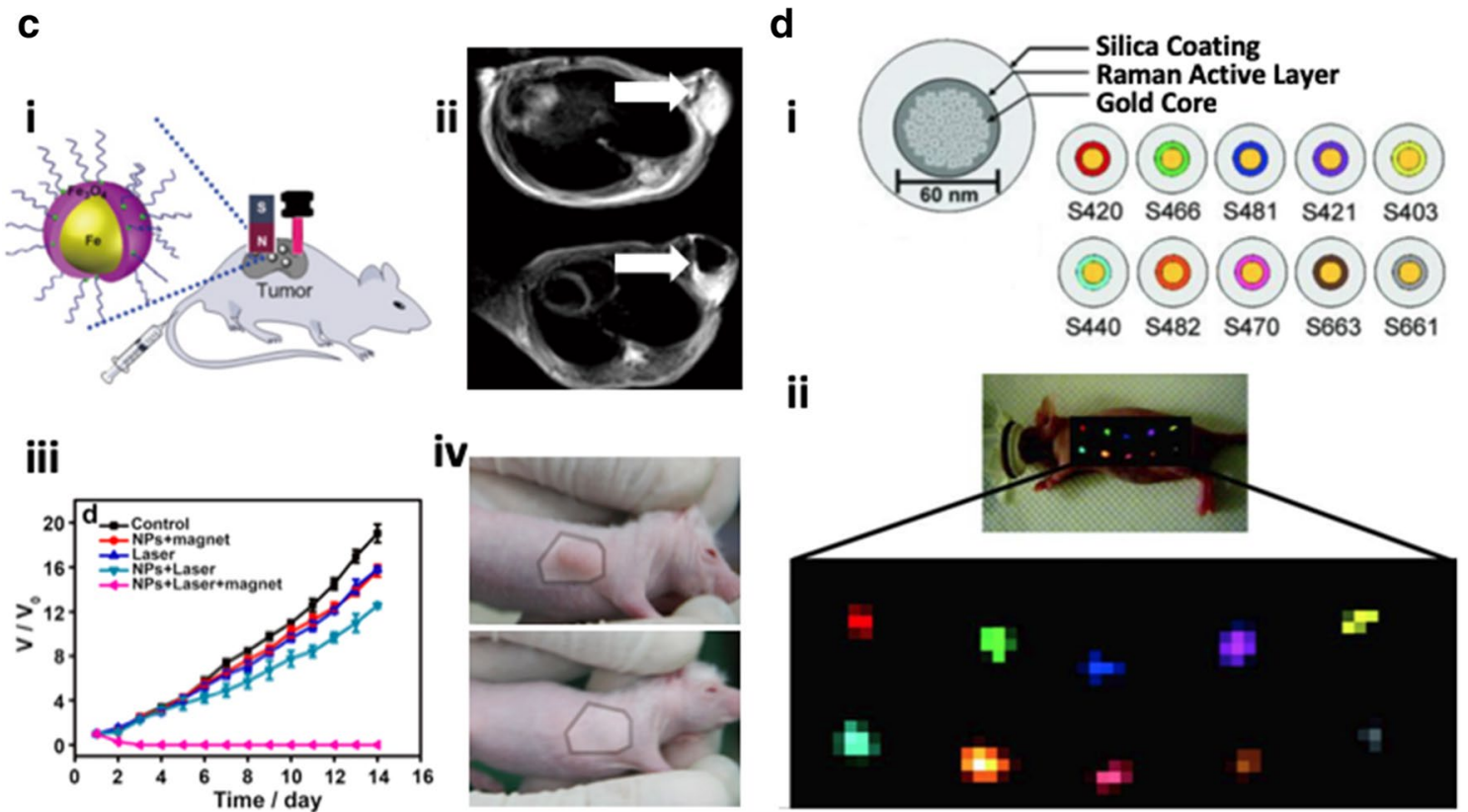

Fig. 1 Class of nanomedicines applied in medical fields. a Nanoliposome used for the delivery of therapeutic drugs or imaging agents. i Schematic illustration of PEGylated liposomes bearing 9AzSia that can be injected i.v. through the tail vein. ii Schematic description of 9AzSia incorporation mechanism into the brain tissue through the binding to sialic acid. iii The liposomes bearing 9AzSia were significantly accumulated in the brain tissue compared to other conditions. Reproduced with permission [36]. Copyright 2016, PNAS. b Polymeric nanoparticles employed for the targeted drug delivery. i Schematic representation of paclitaxel-loaded Angiopep-PEG-PCL nanoparticles. ii Angiopep-conjugation increased targeting efficiency of brain tumors. Reproduced with permission [62]. Copyright 2011, ELSEVIER. c Multimodal iron oxide nanoparticles equipping both MRI and near-infrared PTT techniques. i Cartoon illustration of PEGylated $\mathrm{Fe}_{3} \mathrm{O}_{4}$ nanoparticles along with magnets under the magnetic field or laser irradiation for MRI or PTT. ii MRI images of HeLa bearing tumor mice without (upper) or with magnets (bottom). iii, iv Complete elimination of tumors in mice receiving magnetic targeted PTT. Reproduced with permission [90]. Copyright 2014, ELSEVIER. d Gold nanoparticles achieving multiple colored-imaging. i Schematic of SERS nanoparticles, a gold core coated with a Raman active layer. Depending on the type of Raman layer, the nanoparticles emit different colors. ii Combined images of multiplexing 10 different SERS nanoparticles confirmed in in vivo mice models. Reproduced with permission [104]. Copyright 2009, PNAS

that are similar to the liposome techniques [42]. The exosomes recently received great attentions as they can closely mimic membrane compositions of host cells by presenting the cell-driven surface moieties; thus, they naturally possess potentials to target diseased cells that overcome biological barriers including $\mathrm{BBB}$ and 
Table 2 List of nanomedicines targeting CNS diseases

\begin{tabular}{|c|c|c|c|c|c|c|}
\hline & $\begin{array}{l}\text { Targeting } \\
\text { disease }\end{array}$ & Loading molecule & Purpose & Material & Targeting strategy & Refs. \\
\hline \multirow[t]{7}{*}{ LBNP } & GBM & Doxorubicin, vincristine & Anticancer & Liposome & T7 peptide & {$[11]$} \\
\hline & & Doxorubicin & Anticancer & Liposome & Chlorotoxin & {$[8]$} \\
\hline & & Doxorubicin & Anticancer & Liposome & IL-13 & {$[9]$} \\
\hline & & Coumarin-6 & In vivo imaging & Liposome & RGD, Transferrin & {$[10]$} \\
\hline & $A D$ & Si-BACE & AD treatment & Exosome & Lamp-2b & {$[50]$} \\
\hline & PD & Dopamine $\mathrm{HCl}$ & PD treatment & Liposome & - & {$[30]$} \\
\hline & & L-DOPA & PD treatment & Liposome & Chlorotoxin & {$[31]$} \\
\hline \multirow[t]{11}{*}{ PNP } & GBM & Docetaxel & Anticancer & PEG-PCL & AS14111 aptamer & {$[12]$} \\
\hline & & Paclitaxel & Anticancer & PEG-PLGA & AS14111 aptamer & [13] \\
\hline & & Paclitaxel & Anticancer & PLGA & Transferrin & {$[14]$} \\
\hline & & Paclitaxel & Anticancer & PEG-PLGA & Pep-1 & {$[15]$} \\
\hline & & Paclitaxel & Anticancer & PEG-PCL & Angiopep & {$[62]$} \\
\hline & $A D$ & Curcumin & AD treatment & PBCA & ApoE3 & {$[63]$} \\
\hline & & - & PET & PBCA & ${ }^{125}$-clioquinol & {$[64]$} \\
\hline & $P D$ & a-synuclein & PD treatment & PBCA & ApoE & {$[65]$} \\
\hline & & L-DOPA & PD treatment & PLGA & - & {$[59]$} \\
\hline & & Nicotine & PD treatment & PLGA & - & {$[60]$} \\
\hline & & Ropinirole- $\mathrm{HCl}$ & PD treatment & Chitosan & - & {$[58]$} \\
\hline \multirow[t]{6}{*}{ IO-NP } & GBM & - & MRI & PEG-ION & Chlorotoxin & {$[84]$} \\
\hline & & - & MRI & Dex-SIPON & Chitosan & {$[86]$} \\
\hline & & - & $\mathrm{MRI} / \mathrm{TEM}$ & PEG-ION & Anti-EGFRviii & {$[85]$} \\
\hline & $A D$ & - & MRI & SPION & Anti-AßPP & {$[88]$} \\
\hline & & - & MRI & Dextran-ION & Anti-ferritin & {$[89]$} \\
\hline & PD & Rhodamine-B & MRI & MION & - & [156] \\
\hline \multirow[t]{5}{*}{ Au-NP } & GBM & Phthalocyanine 4 & PDT & PEG-Au-NP & Transferrin peptide & {$[16]$} \\
\hline & & - & PTT & PEG-Au-NP & - & {$[105]$} \\
\hline & & - & PTT & PEG-Au-NP & RVG29 peptide & {$[17]$} \\
\hline & $A D$ & Polyoxometalate & AD treatment & Au-NP & - & [138] \\
\hline & PD & Si-a-synuclein & PD treatment & $\mathrm{Au}-\mathrm{NP}$ & Chitosan & [157] \\
\hline
\end{tabular}

targeted-cellular membranes [43]. One of the outstanding cell membrane-mimicking features of exosomes, which can be applied for CNS drug delivery, is its ability to inherently express antiphagocytic markers (e.g., CD47) that endows the capability to evade monocytes and macrophages of the reticuloendothelial system (RES) reducing immune response and their clearance rate in the blood [44]. Recent studies showed that administration of drug-loaded exosomes to the mouse body or brain had significantly decreased neuroinflammatory activity and reduced septic shock compared to liposaccharide (LPS)-injected mice [45, 46]. Moreover, the enhanced endocytosis, driven by the interactions between targeting receptors on the cells and naturally expressed ligands on the exosomes, could overcome multi-drug resistance by the drug efflux transport pump such as P-glycoprotein (P-gp), which rejects drugs outside of cells and educes drug efficacy [47]. These exosomes have been decorated with active-targeting ligands (iRGD [48, 49], etc.), anchoring molecules (Lamp-2b [50], etc.), and stealth polymers (PEG [51], dextran sulfate [52], etc.) to further boost targeting efficiency, cellular uptake, and circulation half-life. However, the low mechanical stability is still an issue to be solved in order to employ them to nanomedicine platforms for CNS diseases.

\subsection{Polymeric nanoparticles (PNPs)}

Solid polymeric nanoparticles typically represent nanosized and homogeneous spherical structures composed of biocompatible and biodegradable polymers, which encapsulate drugs inside of the particles or attached them to the surface of particles [53]. The polymeric nanocapsule is another form of polymeric nanoparticles having an internal cavity surrounded by polymeric shell that entraps drugs [54]. Encapsulation of drugs is highly affected by interactions between polymer and 
drug molecules. When the polymer-drug interaction is dominant than drug-drug interaction, drugs can be stably incorporated in the polymeric nanoparticles while drug crystallization will happen when the polymer-drug interaction is weaker than drug-drug interaction. To improve the drug encapsulation efficiency in the polymeric nanoparticles, various natural polymers (albumin [55], alginate [56], gelatin [57], chitosan [58], etc.) and synthetic polymers (poly(lactic-co-glycolic acid) (PLGA) [13-15, 59, 60], poly(lactic acid) (PLA) [61], poly(caprolactone) (PCL) [12, 62], poly(butyl cyanoacrylate) (PBCA) [63-65], poly(D,L-lactic acid) (PDLLA) [66], etc.) have been employed and widely applied in medicine and sensor areas. The amphiphilic block copolymers can be employed to form a nanosphere consisting of a hydrophobic solid core with a hydrophilic outer shell. This unique structure not only offers a versatile encapsulation efficiency for both hydrophobic drugs in the core and hydrophilic molecules in the outer shell but also improves the water solubility of nanospheres. Polymeric nanoparticles can be synthesized by several methods depending on the physicochemical characteristics of the compounds [53]. One of the most popular method is either oil-in-water $(\mathrm{o} / \mathrm{w})$ or water-in-oil (w/o) emulsion technique to produce nano-sized oil droplets encapsulating hydrophobic drugs in the water or vice versa [56]. Nanocapsules can be prepared by water-in-oil-in-water $\left(\mathrm{w}_{1} / \mathrm{o} / \mathrm{w}_{2}\right)$ double emulsion technique [66]. The nanoprecipitation, also known as a solvent displacement method, was introduced to control over the size of nanoparticles by maintaining conditions for the dropwise addition of the mixture of polymer and drugs to an aqueous solution forming nano-sized droplets in the water phase [67]. The prominent advantage of solid nanoparticles is a mechanical stability contributing to a long blood circulation time than liposomes [53]. The additional modification with PEG provides stealth property that further increases the half-life of nanoparticles [12, 13, 15, 61]. Another beneficial property is the flexible outer layer that offers multiple conjugating sites for an efficient targeting. For instance, Xin et al. showed that the tailored PCL nanoparticles exhibiting Agiopep, which target lipoprotein receptors (LPRs) on the BBB and glioma cells increasing the drug delivery efficacy, especially for GBMs (Fig. 1b) [62]. Other examples are summarized in Table 2. However, a critical issue for PNPs is a rapid initial release of cargo from the nanoparticles, called a burst effect that attributes to weak interactions of drug to polymer.

\subsection{Magnetic iron oxide nanoparticles (IO-NPs)}

Magnetic nanoparticles have exclusively applied in separation and sensing of biological molecules, targeted gene/drug delivery, clinical diagnosis via magnetic resonance image (MRI), and therapy via magnetic fluid hyperthermia (MFH) [68]. The most widely applied magnetic nanoparticles are iron oxides-based nanoparticles, such as $\mathrm{Fe}_{3} \mathrm{O}_{4}$ (ferrimagnetic, superparamagnetic when the size is less than $15 \mathrm{~nm}$ ), $\alpha-\mathrm{Fe}_{2} \mathrm{O}_{3}$ (hematite, weakly ferromagnetic or antiferromagnetic), $\gamma-\mathrm{Fe}_{2} \mathrm{O}_{3}$ (maghemite, ferrimagnetic), $\mathrm{FeO}$ (wüstite, antiferromagnetic), $\varepsilon-\mathrm{Fe}_{2} \mathrm{O}_{3}$, and $\beta-\mathrm{Fe}_{2} \mathrm{O}_{3} . \mathrm{Fe}_{3} \mathrm{O}_{4}$-based nanoparticles. They have emerged as favorable candidates, particularly for biomedical applications due to their remarkable biocompatibility and hyperparametric characteristics [69]. In order to synthesis the iron oxide nanoparticles with controlled size and shape, high stability, and improved biocompatibility, a number of preparation methods have been proposed in the last decades. The synthesis techniques involve co-precipitation [70, 71], thermal decomposition [72, 73], hydrothermal synthesis [74, 75], microemulsion [76, 77], sonochemical synthesis [78, 79], electrochemical synthesis [80], laser pyrolysis [81], and microorganism-mediated synthesis [82]. Iron oxide nanoparticles have desired mechanical properties besides novel electrical, magnetic, and optical properties. The superparamagnetic nanoparticles are tailored to obtain excellent imaging properties to be used as MRI contrast agents since the MR signal intensity is significantly modulated without any decrease in the T1 and $\mathrm{T} 2$ relaxation times by surrounding water protons [83, 84]. Moreover, magnetic iron oxide nanoparticles have been PEGylated to improve bioavailability and biocompatibility by reducing oxidation and aggregation $[84$, 85]. The carboxylation-polysaccharide coating (dextran [86], ferumoxytol [87], etc.) significantly reduced clearance rate in blood and increased half-life of iron oxide nanoparticles. The surface modification with targeting molecule (chlorotoxin [84], chitosan [86], anti-AßPP [88], anti-ferritin [89], anti-EGFRvIII [85], etc.) allows the increased deposition in the CNS diseases. Moreover, the coating or recruiting the inorganic metallic layer offers additional physicochemical properties, such as magneto-optical properties, magnetic-electrical properties, and magnetic-thermal properties. For instance, the administrated PEGylated $\mathrm{Fe}_{3} \mathrm{O}_{4}$ nanoparticles along with $\mathrm{Nd}-\mathrm{Fe}-\mathrm{B}$ magnet equipping multimodal functions as MRI and near-infrared photothermal therapy (PTT) to successfully achieve the elimination of tumors (Fig. 1c) [90]. Gold coating system provides not only the stability to the NPs preventing oxidation in water but also additional binding sites (thiol group) for biological molecules at the NPs surface [91]. Other advanced metallic nanoparticles are briefly organized in Table 2. Despite such incredible features allowing both sensing and treating CNS diseases, the toxicity found in certain types of neuronal cells is still the major concern [92]. 


\subsection{Metallic gold nanoparticles (Au-NPs)}

Metallic gold nanoparticles have been extensively applied in the wide range of biomedical science and engineering areas. These nanoparticles have been intensively applied in diagnostic imaging systems, where most states have been described as the metallic nanoparticle-mediated imaging modalities by using MRI, computed tomography (CT) scan, Positron-emission tomography (PET), ultrasound, and surface-enhanced Raman spectroscopy (SERS) imaging systems nowadays [93]. The most popular method to synthesis gold nanoparticles is the citrate reduction of gold tetra-chloric acid, which is low-cost and performed under mild preparation environments [94]. Other preparation methods include chemical reduction $[95,96]$, photochemical synthesis using UV irradiation [97, 98], sonochemical synthesis [99], laser ablation [100], seed mediated growth [101], and sonoelectrochemical synthesis [102]. Gold nanoparticles especially have received great attentions as promising biomaterials in bio-nanotechnology and related areas due to their unique optical and thermal properties as well as ease of surface functionalization, which enable targeted delivery of genes/proteins/drugs and various biological assays. The optical property mostly attributes to the localized surface plasmon resonance (LSPR), which absorb and emit various light colors depending on size, shape, local reflective index, and aggregate status of nanoparticles (Fig. 1d) $[103,104]$. As increasing in the aspect ratio forming gold nanorods, they can absorb from visible up to Infra-red region and obtain increased oscillator strength, which allows PTT. Numerous studies have developed gold nanoparticles functionalized with targeting molecules for brain cancers and BBB and applied them for the diagnosis and treating purposes. Especially, the targeted gold nanoparticles are beneficial for the PTT ablation of brain tumors; upon reaching to the brain tumor parts, the laser light at the IR region can pass through the skin, warm-up the tumor part, and specifically kill the cancer while sparing healthy tissues $[16,17,105]$. Recently, gold nanoshells and nanocages are developed for further application to high-resolution imaging techniques such as MRI [69]. Currently developed gold nanoparticles for the CNS diseases are summarized in Table 2. However, the longterm cytotoxicity is a major limitation to be solved in the future [106].

\section{Promising strategies to improve drug penetration into $\mathrm{BBB}$}

The blood-brain barrier is a tighten mechanical barrier between blood streams and brain tissues, which is highly selective and only permits the entrance of essential water, nutrients, and neurotransmitters governing the maintenance of CNS homeostasis and modulation of neuronal signal propagation [5]. Moreover, this barrier regulates the immune cell transports to maintain the intracranial pressure but limit the entry of toxins and pathogens circulating in the blood to prevent potential neuronal damages. The presence of this unique and restrictive barrier is the major limitation to deliver therapeutic drugs or imaging compounds to CNS regions. This barrier has known to reject the entrance of $98 \%$ of small-molecule drugs and $\sim 100 \%$ of large-molecule drugs to the CNS [107]. To achieve a successful delivery of nanomedicines to the CNS across the BBB, a number of recent studies have investigated to understand the characteristics of BBB transports and adopt the mechanisms on the design of nanomedicines. There are five major pathways to permit the entrance of molecules across the BBB: (1) paracellular pathway through tight junctions for hydrophilic molecules, (2) transcellular diffusive pathway for hydrophobic molecules, (3) transporters-mediated specific molecules (4) receptors-mediated transcytosis of ligands or ligand-conjugated carriers, and (5) absorption of positive charged molecules [5]. Among the pathways, most nanomedicines select strategies of encapsulating drugs in the adsorptive molecules promoting passive transports or nanomedicines conjugated with transporters or receptors improving active transports across the BBB (Fig. 2a) [5].

\subsection{Passive transporting system}

The electrostatic interaction-mediated adsorption is a passive diffusion for small biomolecules driven by a concentration gradient across the BBB [5]. One of novel molecules promoting the passive diffusion across the $\mathrm{BBB}$ is albumin. Albumin is an abundant component of human blood and has been widely applied in the medical fields as a novel biomaterial due to their excellent biocompatibility, long half-life in blood ( 20 days), and ability to pass across the BBB [108]. Moreover, the free thiol groups on albumin can offer binding sites for metal ions allowing efficient incorporation with metal nanoparticles by coating the metal surface (Fig. 2c) [109]. The albumin is especially beneficial for brain cancer treatments because of albumin binding receptors, such as SPARC and gp60, overexpressed on gliomas [55]. Various albumin-based nanomedicines have been clinically approved for drug carrier platforms targeting various diseases (e.g., diabetes, various tumors, multiple sclerosis, etc.) and precise diagnosis or bioimaging systems (e.g., SPECT, PET, MRI, ultrasound imaging, etc.) [110]. In order to apply them for the CNS diseases, cationic albumin-coated PEG-PLA nanoparticles were designed to promote albumin-mediated passive transport across $\mathrm{BBB}$ showing 7.9-fold increased BBB transport efficiency compared to non-charged nanoparticles [61]. However, this passive 


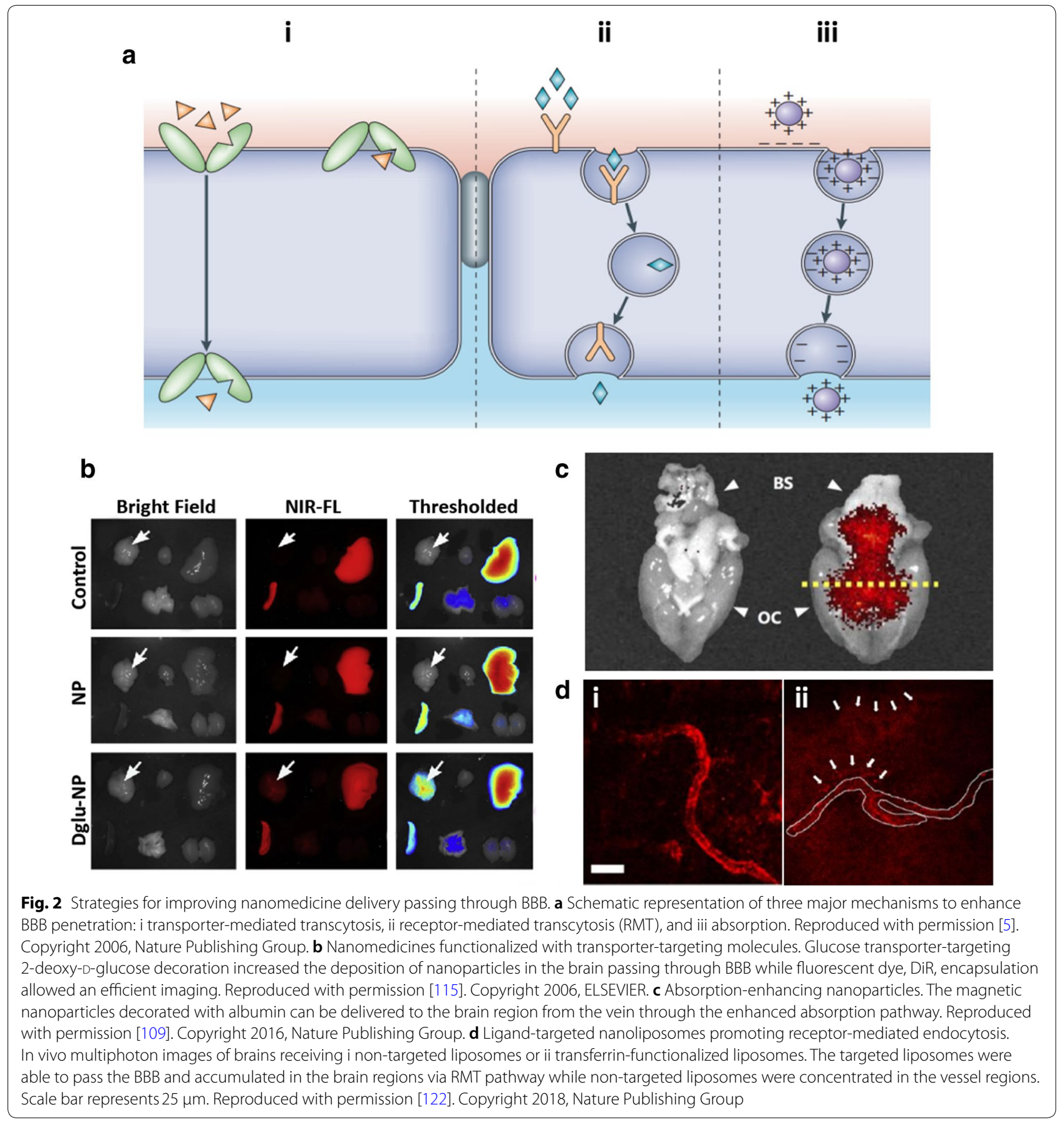

diffusion transport system has not been solely applied in the development of nanomedicines targeting the CNS, but combined with other passive or active targeting systems due to the low targeting efficiency. The albuminbased nanoparticles have been decorated with targeting antibodies against receptors expressed on brain endothelial cells such as transferrin and insulin [111, 112]. Moreover, Lin et al. improved in vitro BBB penetration of albumin nanoparticles by modification of albumin with a cell-penetrating peptide, low molecular weight protamine (LMWP), and applied the nanoparticles to co-encapsulate anticancer drugs, paclitaxel and fenretinide, and deliver them to the CNS region [55]. This study reported that LMWP-albumin nanoparticles achieved 2.5-fold increased BBB penetration compared to bare albumin nanoparticles. 
Another passive transporting system can be promoted by decorating transport protein on the surface of nanoparticles [5]. Transport proteins, expressed on the endothelial cells in both the abluminal and luminal sides, modulate the influx of essential nutrients, peptides, and ions or the efflux of wastes and toxins. For instance, glucose transporters (GLUTs), highly expressed on endothelial cells, mediate the influx of sugars to the CNS by facilitated diffusion in response to the concentration gradient due to high demands on glucose and other energy sources (e.g., mannose, glucosamine, fructose, and other glucose derivatives) in the brain [113]. In this regard, the conjugation of glucose or glucose analogues, specifically targeting type 1 transporter (GLUT1), has widely applied in the nanomedicines to enhance transports across the BBB. For instance, $\beta 2$-mercaptoethoxyglucose-conjugated gold nanoparticles have proven at least 3-times higher potentials to pass the 3D cultured BBB models with hCMEC/D3 cells through binding to GLUT1, compared to glutathione-conjugated gold nanoparticles [114]. Moreover, gold nanoparticles decorated with 2-deoxy-D-glucose, which has known to increase the BBB penetration efficiency through interactions with GLUT1 (Fig. 2b) [115], produce a high-resolution and anatomic information in a single CT scan of tumors [116, 117]. However, a number of studies reported that glycosylation frequently failed to promote the BBB penetration due to random conjugation of sugars to the nanomedicines since $\mathrm{OH}$ groups only positioned at $\mathrm{C} 1, \mathrm{C} 3$, and $\mathrm{C} 4$ of glucose and its derivatives are actual binding sites of GLUT1 [118]. To increase transcytosis of glycosylated nanomedicines across the $\mathrm{BBB}$, Anraku et al. designed PEG-micelles conjugated with $\mathrm{C} 6 \mathrm{OH}$ group in order to preserve the binding ability of glucose to GLUT1 [119]. The results showed that the linkage at C6 improved interactions with GLUT1 and the significantly increased BBB penetration rate compared to the linkage at C3.

\subsection{Active transporting system}

The transporting system based on the receptor-mediated transcytosis (RMT) is the most efficient and promising strategy to promote BBB permeabilization [5]. The receptors expressing on the luminal side of brain endothelial cells and mediating RMT, include transferrin receptors (TRs), scavenger receptors (SRs), insulin receptors (IRs), and lipoprotein receptors (LPRs). In contrast to passive transport across the $\mathrm{BBB}$, the RMT is an active transport against concentration gradient requiring large amount of energy [120]. Upon the binding of ligands on their receptors, ligands and ligand-decorated molecules/carriers undergo endocytosis via membrane invagination forming intracellular transport vesicles. The vesicles containing ligand-receptor complex are experiencing exocytosis that transports the vesicles to the basolateral side so that the vesicles are fusing with the plasma membrane and releasing the encapsulated components to the CNS. Due to the tightly regulated process of the RMT, nanomedicines conjugated with ligands initiating the RMT offers ideal platforms for selective delivery of cargo to the CNS compared to passive transporting systems. Among the ligands or antibodies targeting receptors expressed on the endothelial cells, the transferrin peptide, transferrin protein, and antibody against transferrin (anti-TRs) have been tremendously explored in terms of their ability to promote the RMT across the BBB (Fig. 2d) [121, 122]. Not only the high binding affinity to TRs but also the avidity effect, TR-targeting system has been deemed a promising strategy to enhance the RMTmediated transport into brain parenchyma from systemic administration [123]. In this regards, a number of studies conjugated transferrin peptides or anti-TRs to various nanomedicine platforms, such as human serum albumin (HSA) nanoparticles [111], gold nanoparticles [123], and nanoliposomes [124], and proved the promoted the BBB penetration of nanomedicines. Another evident strategy is targeting IRs to promote the $\mathrm{BBB}$ transport. A number of studies employed antibodies against IRs (anti-IRs) instead of insulin or insulin peptides due to their notable targeting efficiency. Coloma et al. combined the anti-IRs with glial-derived neurotrophic factor peptide (GDNF), which showed great potentials to target both neurons and the BBB [125]. Ulbrich et al. decorated HSA nanoparticles with anti-IRs (29B4) and reported the improved drug delivery efficacy across the BBB [112]. To further increase the $\mathrm{BBB}$ penetration, nanomedicines can be tailored with dual ligand-conjugation system with two types of ligands, one for initiating transcytosis of nanomedicines across the BBB and the other for further promoting penetration mechanisms.

\section{Prospective approaches to enhance the targeted delivery to diseased regions}

For the successful drug delivery, nanomedicines should be delivered to the specific regions around the targeted diseases [126, 127], in order to reduce unexpected cytotoxicity in the brain where most of cells governing brain functions and immune systems can be easily disrupted by external molecules. Both passive and active targeting systems can be applied for the development of CNS disease-targeting nanomedicines; however, the passive targeting system is not solely adopted excepting for the brain tumor-targeting nanomedicines. Therefore, several studies employed the combined targeting systems in order to achieve both accurate diagnosis and treatment for the specific diseased brains. 


\subsection{GBM-targeting system}

Passive targeting systems have been applied in the design of brain cancer-targeting nanomedicines due to the highly leaky vasculature environments around the tumors. The enhanced permeation and retention (EPR) is the unique phenomenon that allows nanomedicines loading therapeutic drugs with size range of 150-200 nm to be easily accumulated in the tumor regions passing through the vasculatures having several pores with size range of 200-500 $\mathrm{nm}$ [7]. The nanoparticles, equipped with the property of inducing EPR effects by controlling size below $200 \mathrm{~nm}$, not only enhance the penetration to the BBB but also increase the accumulation of drugs in the solid tumors more than several-fold higher compared to free drugs. In addition, these nanoparticles are advantageous for the drug delivery using interventional procedures such as catheters. The anticancer drugs encapsulated in liposomes (Doxil1/Caelyx1, Myocet1, Daunosome1 loading doxorubicin) or polymeric nanoparticles (Abraxane encapsulating paclitaxel) are particularly designed to promote the EPR effects and recently received clinical approvals [128]. Despite the enhanced penetration toward the solid tumors, more than $95 \%$ of passively targeted nanomedicines failed to deliver their cargo to the brain tumor regions, even after multiple administration trials through the intravenous methods causing unexpected side effects such as neuroinflammation and cytotoxicity in the brain [129]. Thus, the passive targeting strategy has to be combined with another targeting strategy, such as active targeting system with tumor-specific targeting ligands [12, 130].

Another brain tumor-targeting strategy is the active targeting system by decorating nanomedicines with targeting ligands, which have high binding affinity to receptors or other surface membrane proteins overexpressed on targeting brain tumors [128]. The ligands include small molecules, peptides/aptamers, proteins, and antibodies as well as their fragments that specifically bind to the receptors and trigger the internalization of the decorated nanomedicines. The major advantage of the active targeting over the passive targeting is an incredible selectiveness to the specific tumors that enable the effective delivery of therapeutic drugs or diagnostic reagents to disease sites reducing local cytotoxicity [131]. As previously described, the transferrin, a gold standard targeting molecule for increasing the BBB penetration, and its moieties (e.g., TfR-lytic hybrid peptide [132], OX26 [111], anti-TR antibodies $[15,90]$, etc.) have been extensively established as brain targeting molecules as well and decorated on various nanomedicines. In addition, the conjugation of LDLR, LRP, or IR targeting molecules on the nanomedicines have great potentials to enhance the $\mathrm{BBB}$ penetration as well as internalization into brain tumors
$[112,133,134]$. The hyaluronic acid (HA) and folate, two general tumor targeting molecules as they have high affinities to CD44 and folate receptors that are highly expressed on various cancer cells including brain tumors, have proven their excellent targeting efficiency of HA or folate-decorated nanomedicines to brain tumors compared to bare nanomedicines (Fig. 3a) $[135,136]$. Other verified ligands to promote cell uptake by brain tumors, are IL-13 [9], RGD [130], and mutant receptors expressed on the GBMs (e.g., EGFRviii) [85]. In spite of excellent delivery efficiency, the high heterogeneity found in most brain tumors reduces the therapeutic efficacy of targeted nanomedicines [137]. To overcome such limitation, currently developed drug delivery carriers have been modified with multiple targeting molecules. The dual ligand-conjugation system with two targeting machineries can improve both penetration of nanomedicines across BBB and accumulation in brain tumors $[12,130]$.

\subsection{Alzheimer's disease-targeting system}

As described in the previous section, deposition of amyloid-beta $(\mathrm{A} \beta)$ peptides around $\mathrm{AD}$ neurons is the key signature of $\mathrm{AD}$ progression [20]. In order to deliver nanomedicines to $\mathrm{AD}$ brains, the nanoparticles have been decorated with ligands targeting either $\mathrm{A} \beta$ oligomers/plaques or associated modulators [64, 88, 89, 138]. Through the ligand-decoration, the nanomedicines can achieve the local delivery of $\mathrm{AD}$ treatments or imaging reagents to the disease regions surrounded by $A \beta$ while sparing healthy brain parts. For the diagnosis of early $A D$ via MRI, the recently developed gadolinium (Gd)-based nanoparticles having excellent imaging contrast efficiency, were conjugated with various $A \beta$ aggregate-targeting molecule such as popular $A \beta$ probe (e.g., Pittsburgh compound B [139], etc.) and anti-A $\beta$ antibody (e.g., IgG4.1 [140], LPFFD [141], KLVFF [141], etc.) (Fig. 3b). Furthermore, apolipoprotein E3, a high ADrisk marker associating with $\mathrm{A} \beta$ depositions, has proven its targeting efficiency for $\mathrm{AD}$ brains, and recent study introduced ApoE3-functionalized PBCA nanoparticles to achieve the enhanced uptake by AD brains [63]. For the efficient delivery of therapeutic drugs to AD brains, Zhang et al. developed the PEG-PLA nanoparticles with TGN peptides for the enhanced $\mathrm{BBB}$ penetration and QSH peptides for the A 342 -binding, which achieved 3.41-fold increased targeting efficiency for AD compared to bare nanoparticles [142]. However, A $\beta$-targeting system limits to detect and treat for early staged AD brains, indicating the significance of developing new targeting systems for the AD models in later stages. Therefore, nanomedicines conjugated with multiple check points involved in $\mathrm{AD}$ pathogenesis would address the current limitations found in $\mathrm{AD}$ treatments. In addition, present 

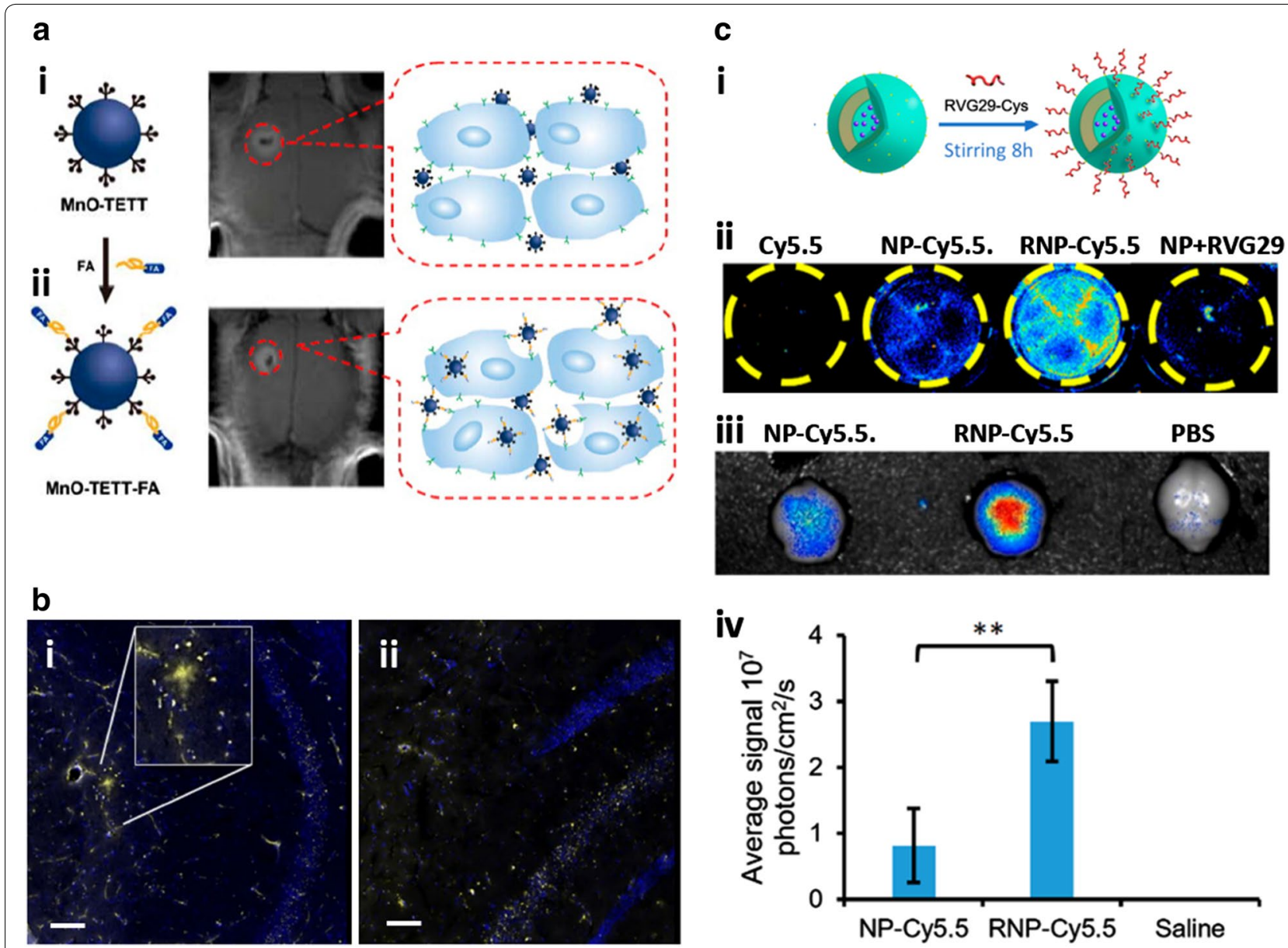

Fig. 3 Approaches for targeting each CNS disease. a Nanomedicines functionalized with ligands targeting receptors expressed on GBMs. The oleic acid capped manganese oxide ( $\mathrm{MnO}$ ) oxide nanoparticles were decorated with folic acid, a glioma-specific moiety. MRI images of brains bearing C6 tumors, receiving i MnO NPs decorated with N-(trimethoxysilylpropyl) ethylene diamine triacetic acid (TETT) silane or ii MnO NPs decorated with both TETT and folic acid via post intravenous injection for $2 \mathrm{~h}$, confirmed the deposition of targeted nanoparticles in the small tumor region. Reproduced with permission [115]. Copyright 2014, American Chemical Society Publications. b Aß-targeted nanomedicines for AD diagnosis. MRI images show that i Gadolinium (Gd)-based nanoparticles decorated with $A \beta$-targeting peptides were accumulated around $A \beta$ plaques in the $A D$ mouse brain while ii bare $\mathrm{Gd}$ nanoparticles were not able to detect A $\beta$ plaques. Scale bars represent $50 \mu \mathrm{m}$. Reproduced with permission [141]. Copyright 2016, Springer Nature. c Nanomedicines targeting PD. i Schematic configuration of PD-targeted nanoparticles. The conjugation of RVG29 to nanoparticles (RNP-cy5.5) achieved, ii the significantly enhanced in vitro BBB permeability and the iii, iv effective delivery to in vivo PD brain region compared to bare nanoparticles. Reproduced with permission [148]. Copyright 2018, American Chemical Society Publications

sensing and analytic nanoplatforms can further contribute to the discovery of novel markers targeting late-onset AD models.

\subsection{Parkinson diseases-targeting system}

For the diagnosis purpose, the detection of decreased dopamine and increased acetylcholine levels in the brain tissue, bloods, urine samples are the major strategies to diagnose PD $[25,143]$. To this end, the receptors or aptamers having the high affinity to the neurotransmitters are conjugated with conductive nanomedicines, which are coated on the electrode. Bardon et al. showed gold nanoparticles cross-linked with biscyclo (paraquat-p-phenylene) receptors to detect dopamine and L-DOPA achieving a detection limit of $1 \times 10^{-6}$ $M$ [144]. Recent studies applied the dopamine-binding aptamer-coated gold nanoparticles immobilized on the electrode to further improve the sensitivity up to $1 \times 10^{-8}$ $\mathrm{M}$, which allow to detect the decreased dopamine level from urine samples with high accuracy [145]. However, the current sensing platforms based on electrochemical property of dopamine binding to the receptors or aptamers require several steps and longer time compared to imaging techniques, such as MRI, CT, and etc.

The major strategy for the delivery of dopamine or other therapeutic molecules/DNA to promote neural 
viability in the $\mathrm{PD}$ area is improving the $\mathrm{BBB}$ penetration by decorating nanoparticles with transferrin or lactoferrin, which results in the accumulation of drugs in the CNS region [146]. To further target neurons, neurotransmitters binding to the dopamine D2 and D3 receptors have been proposed as useful ligands for targeting PD brains [25]. In addition, rabies virus glycoprotein (RVG) peptide targeting acetylcholine receptors and Tet1 peptide targeting GT1b gangliosides highly expressed on neuronal cells, can be used for improving the entry pathway into neurons [147]. You et al. described polymeric nanoparticles conjugated with RVG peptides and proved their great potentials to deliver the PD preventing drugs to neurons by using PD mouse models (Fig. 3c) [148]. Since there are no representative biomarkers expressed on the PD brains, the current PD-targeting nanomedicines cannot target the disease specific regions causing unexpected toxicity in the CNS. Therefore, it is an urgent issue to discover novel biomarkers highly expressed on PD neurons so that improve the targeted delivery of PD drugs or PD detecting reagents encapsulated in the nanomedicines to the disease brains.

\section{Conclusion and outlook}

To sum up, we overviewed the currently developed nanoplatforms for prognosis purpose as well as the promising strategies for the targeted delivery of drugs to the disordered CNS regions effective for GBM, AD, and PD. Through the enormous efforts to discover biomarkers and create nanomedicines targeting the biomarkers in the past decade, a number of studies have made fruitful progress to overcome current limitations that retard the clinical translation of CNS-targeting medicines. Moreover, recently proposed hybrid nanomedicines, combined with two or more nanomedicines, have accomplished diverse functions, such as dual or multiple targeting systems, multimodal therapies, multimodal diagnosis, or their combinations. These studies have thoroughly proven the incredible abilities of hybrid nanomedicines for detecting or treating neurological disorders both in vitro and in vivo, which may lead to success on the clinical translation at the end. Therefore, the significant improvement in the nanotechnologies for synthesis and functionalization of versatile nanomedicines will contribute to the discovery of novel biomarkers and nanomedicines incorporated with the biomarkers to bring promising strategies to conquer the CNS diseases in the near future.

\section{Authors' contributions}

YJK, EGC, HC reviewed literature, outlined, wrote, edited the manuscript and prepared figures. All authors read and approved the final manuscript.
Completing interests

The authors declare that they have no competing interests.

Availability of data and materials

Not applicable.

Funding

This research was supported by Pioneering Funding Award funded by Cure Alzheimer's Fund (CAF, HC).

\section{Publisher's Note}

Springer Nature remains neutral with regard to jurisdictional claims in published maps and institutional affiliations.

Received: 15 October 2018 Accepted: 20 November 2018

Published online: 30 November 2018

References

1. M. Koshy et al., Improved survival time trends for glioblastoma using the SEER 17 population-based registries. J. Neurooncol. 107, 207-212 (2012)

2. J. Wang, B.J. Gu, C.L. Masters, Y.-J. Wang, A systemic view of Alzheimer disease-insights from amyloid- $\beta$ metabolism beyond the brain. Nat. Rev. Neurol. 13,612 (2017)

3. W. Poewe et al., Parkinson disease. Nat. Rev. Dis. Primers 3, 17013 (2017)

4. J. Cummings et al., Agitation in cognitive disorders: International Psychogeriatric Association provisional consensus clinical and research definition. Int. Psychogeriat. 27, 7-17 (2015)

5. N.J. Abbott, L. Rönnbäck, E. Hansson, Astrocyte-endothelial interactions at the blood-brain barrier. Nat. Rev. Neurosci. 7, 41 (2006)

6. W.H. De Jong, P.J.A. Borm, Drug delivery and nanoparticles: applications and hazards. Int. J. Nanomed. 3, 133-149 (2008)

7. K. Greish, Cancer nanotechnology: methods and protocols (eds Stephen R. Grobmyer \& Brij M. Moudgil) (Humana Press, New York, 2010), pp. 25-37

8. H. Mahmud et al., Targeting glioblastoma cells expressing CD44 with liposomes encapsulating doxorubicin and displaying Chlorotoxin-lgG Fc fusion protein. Int. J. Mol. Sci. 19, 659 (2018)

9. A.B. Madhankumar, B. Slagle-Webb, A. Mintz, J.M. Sheehan, J.R. Connor, Interleukin-13 receptor-targeted nanovesicles are a potential therapy for glioblastoma multiforme. Mol. Cancer Ther. 5, 3162-3169 (2006)

10. L.I. Qin et al., A dual-targeting liposome conjugated with transferrin and arginine-glycine-aspartic acid peptide for glioma-targeting therapy. Oncol. Lett. 8, 2000-2006 (2014)

11. Y. Zhang et al., Dual-modified liposome codelivery of doxorubicin and vincristine improve targeting and therapeutic efficacy of glioma. Drug Deliv. 24, 1045-1055 (2017)

12. H. Gao et al., Precise glioma targeting of and penetration by aptamer and peptide dual-functioned nanoparticles. Biomaterials 33, 5115$5123(2012)$

13. J. Guo et al., Aptamer-functionalized PEG-PLGA nanoparticles for enhanced anti-glioma drug delivery. Biomaterials 32, 8010-8020 (2011)

14. N. Shah, K. Chaudhari, P. Dantuluri, R.S.R. Murthy, S. Das, Paclitaxelloaded PLGA nanoparticles surface modified with transferrin and Pluronic ${ }^{\circledR}$ P 85 , an in vitro cell line and in vivo biodistribution studies on rat model. J. Drug Target. 17, 533-542 (2009)

15. B. Wang et al., Improved anti-glioblastoma efficacy by IL-13Ra2 mediated copolymer nanoparticles loaded with paclitaxel. Sci. Rep. 5, 16589 (2015)

16. S. Dixit et al., Transferrin receptor-targeted theranostic gold nanoparticles for photosensitizer delivery in brain tumors. Nanoscale $\mathbf{7}$ 1782-1790 (2015)

17. C. Lee et al., Rabies virus-inspired silica-coated gold nanorods as a photothermal therapeutic platform for treating brain tumors. Adv. Mater. 29, 1605563 (2017)

18. B. Huang et al., Advances in immunotherapy for glioblastoma multiforme. J. Immunol. Res. 2017, 3597613 (2017) 
19. A. Kumar, A. Singh, Ekavali, A review on Alzheimer's disease pathophysiology and its management: an update. Pharmacol. Rep. 67, 195-203 (2015)

20. G. Neuroinflammation Working et al., Inflammation and Alzheimer's disease. Neurobiol. Aging 21, 383-421 (2000)

21. P.S. Aisen et al., Tramiprosate in mild-to-moderate Alzheimer's diseasea randomized, double-blind, placebo-controlled, multi-centre study (the Alphase Study). Arch. Med. Sci. 7, 102-111 (2011)

22. M. Janusz, A. Zablocka, Colostral proline-rich polypeptides-immunoregulatory properties and prospects of therapeutic use in Alzheimers disease. Curr. Alzheimer Res. 7, 323-333 (2010)

23. L.E. Rojo, J. Alzate-Morales, I.N. Saavedra, P. Davies, R.B. Maccioni, Selective interaction of lansoprazole and astemizole with tau polymers: potential new clinical use in diagnosis of Alzheimer's disease. J. Alzheimer's Dis. 19, 573-589 (2010)

24. R. Kandimalla, P.H. Reddy, Therapeutics of neurotransmitters in Alzheimer's disease. J. Alzheimer's Dis. 57, 1049-1069 (2017)

25. J.G. Nutt, J.H. Carter, G.J. Sexton, The dopamine transporter: importance in Parkinson's disease. Ann. Neurol. 55, 766-773 (2004)

26. M.G. Spillantini et al., a-Synuclein in Lewy bodies. Nature $\mathbf{3 8 8}, 839$ (1997)

27. S. Md, S. Haque, J.K. Sahni, S. Baboota, J. Ali, New non-oral drug delivery systems for Parkinson's disease treatment. Exp. Opin. Drug Deliv. 8, 359-374 (2011)

28. Y. Agid et al., Tolcapone, bromocriptine, and Parkinson's disease. Lancet 350, 712-713 (1997)

29. B. Thanvi, N. Lo, T. Robinson, Levodopa-induced dyskinesia in Parkinson's disease: clinical features, pathogenesis, prevention and treatment. Postgrad. Med. J. 83, 384-388 (2007)

30. N.K. Jain, A.C. Rana, S.K. Jain, Brain drug delivery system bearing dopamine hydrochloride for effective management of Parkinsonism. Drug Dev. Ind. Pharm. 24, 671-675 (1998)

31. Y. Xiang et al., Chlorotoxin-modified stealth liposomes encapsulating levodopa for the targeting delivery against the Parkinson's disease in the MPTP-induced mice model. J. Drug Target. 20, 67-75 (2012)

32. H. Zhang, Liposomes: methods and protocols (ed Gerard G. M. D'Souza) (Springer, New York, 2017), pp. 17-22

33. F. Olson, C.A. Hunt, F.C. Szoka, W.J. Vail, D. Papahadjopoulos, Preparation of liposomes of defined size distribution by extrusion through polycarbonate membranes. Biochimica et Biophysica Acta Biomembranes 557, 9-23 (1979)

34. B. Yu, RJ. Lee, LJ. Lee, Microfluidic methods for production of liposomes. Methods Enzymol. 465, 129-141 (2009)

35. T.M. Allen, P.R. Cullis, Liposomal drug delivery systems: from concept to clinical applications. Adv. Drug Deliv. Rev. 65, 36-48 (2013)

36. R. Xie et al., In vivo metabolic labeling of sialoglycans in the mouse brain by using a liposome-assisted bioorthogonal reporter strategy. Proc. Natl. Acad. Sci. 113, 5173 (2016)

37. D. Pain, P.K. Das, P. Ghosh, B.K. Bachhawat, Increased circulatory half-life of liposomes after conjunction with dextran. J. Biosci. 6, 811-816 (1984)

38. J.E. Adrian et al., A novel lipid-based drug carrier targeted to the nonparenchymal cells, including hepatic stellate cells, in the fibrotic livers of bile duct ligated rats. Biochimica et Biophysica Acta Biomembranes 1768, 1430-1439 (2007)

39. M. Gary-Bobo, P. Nirdé, A. Jeanjean, A. Morère, M. Garcia, Mannose 6-phosphate receptor targeting and its applications in human diseases. Curr. Med. Chem. 14, 2945-2953 (2007)

40. M. Delač, H. Motaln, H. Ulrich, T.T. Lah, Aptamer for imaging and therapeutic targeting of brain tumor glioblastoma. Cytometry Part A 87, 806-816 (2015)

41. L. Sercombe et al., Advances and challenges of liposome assisted drug delivery. Front. Pharmacol. 6, 286 (2015)

42. R.J. Lobb et al., Optimized exosome isolation protocol for cell culture supernatant and human plasma. J. Extracell. Vesicles (2015). https://doi. org/10.3402/jev.v3404.27031

43. D. Ha, N. Yang, V. Nadithe, Exosomes as therapeutic drug carriers and delivery vehicles across biological membranes: current perspectives and future challenges. Acta Pharmaceutica Sinica B 6, 287-296 (2016)

44. S. Kamerkar et al., Exosomes facilitate therapeutic targeting of oncogenic KRAS in pancreatic cancer. Nature 546, 498 (2017)
45. X. Zhuang et al., Treatment of brain inflammatory diseases by delivering exosome encapsulated anti-inflammatory drugs from the nasal region to the brain. Mol. Ther. 19, 1769-1779 (2011)

46. D. Sun et al., A novel nanoparticle drug delivery system: the antiinflammatory activity of curcumin is enhanced when encapsulated in exosomes. Mol. Ther. 18, 1606-1614 (2010)

47. M.S. Kim et al., Development of exosome-encapsulated paclitaxel to overcome MDR in cancer cells. Nanomed. Nanotechnol. Biol. Med. 12, 655-664 (2016)

48. J. Wang et al., The use of RGD-engineered exosomes for enhanced targeting ability and synergistic therapy toward angiogenesis. Nanoscale 9, 15598-15605 (2017)

49. X. Luan et al., Engineering exosomes as refined biological nanoplatforms for drug delivery. Acta Pharmacol. Sin. 38, 754 (2017)

50. D. Sun et al., Exosomes are endogenous nanoparticles that can deliver biological information between cells. Adv. Drug Deliv. Rev. 65, 342-347 (2013)

51. M.A. Rider, S.N. Hurwitz, D.G. Meckes Jr., ExtraPEG: a polyethylene glycol-based method for enrichment of extracellular vesicles. Sci. Rep. 6, 23978 (2016)

52. D.C. Watson et al., Efficient production and enhanced tumor delivery of engineered extracellular vesicles. Biomaterials 105, 195-205 (2016)

53. A. Nasir, A. Kausar, A. Younus, A review on preparation, properties and applications of polymeric nanoparticle-based materials. PolymerPlastics Technol. Eng. 54, 325-341 (2015)

54. P. Kothamasu et al., Nanocapsules: the weapons for novel drug delivery systems. Biolmpacts 2,71-81 (2012)

55. T. Lin et al., Blood-brain-barrier-penetrating albumin nanoparticles for biomimetic drug delivery via albumin-binding protein pathways for antiglioma therapy. ACS Nano 10, 9999-10012 (2016)

56. A.H.E. Machado et al., Preparation of calcium alginate nanoparticles using water-in-oil (W/O) nanoemulsions. Langmuir 28, 4131-4141 (2012)

57. N. Sahoo, R.K. Sahoo, N. Biswas, A. Guha, K. Kuotsu, Recent advancement of gelatin nanoparticles in drug and vaccine delivery. Int. J. Biol. Macromol. 81, 317-331 (2015)

58. O. Jafarieh et al., Design, characterization, and evaluation of intranasal delivery of ropinirole-loaded mucoadhesive nanoparticles for brain targeting. Drug Dev. Ind. Pharm. 41, 1674-1681 (2015)

59. P.Y. Gambaryan, I.G. Kondrasheva, E.S. Severin, A.A. Guseva, A.A. Kamensky, Increasing the efficiency of Parkinson's disease treatment using a poly(lactic-co-glycolic acid) (PLGA) based L-DOPA delivery system. Exp. Neurobiol. 23, 246-252 (2014)

60. M.N. Tiwari et al., Nicotine-encapsulated poly(lactic-co-glycolic) acid nanoparticles improve neuroprotective efficacy against MPTP-induced parkinsonism. Free Radical Biol. Med. 65, 704-718 (2013)

61. W. Lu, Y.-Z. Tan, K.-L. Hu, X.-G. Jiang, Cationic albumin conjugated pegylated nanoparticle with its transcytosis ability and little toxicity against blood-brain barrier. Int. J. Pharm. 295, 247-260 (2005)

62. H. Xin et al., Angiopep-conjugated poly(ethylene glycol)-co-poly( $\varepsilon$ caprolactone) nanoparticles as dual-targeting drug delivery system for brain glioma. Biomaterials 32, 4293-4305 (2011)

63. R.S. Mulik, J. Mönkkönen, R.O. Juvonen, K.R. Mahadik, A.R. Paradkar, ApoE3 mediated poly(butyl) cyanoacrylate nanoparticles containing curcumin: study of enhanced activity of curcumin against beta amyloid induced cytotoxicity using in vitro cell culture model. Mol. Pharm. 7, 815-825 (2010)

64. P.V. Kulkarni et al., Quinoline-n-butylcyanoacrylate-based nanoparticles for brain targeting for the diagnosis of Alzheimer's disease. Nanomed. Nanobiotechnol. 2, 35-47 (2010)

65. L. Hasadsri, J. Kreuter, H. Hattori, T. Iwasaki, J.M. George, Functional protein delivery into neurons using polymeric nanoparticles. J. Biol. Chem. 284, 6972-6981 (2009)

66. L. Jie, Q. Zhiye, W. Shenqi, Z. Lei, Z. Shengmin, A modified double-emulsion method for the preparation of daunorubicin-loaded polymeric nanoparticle with enhanced in vitro anti-tumor activity. Biomed. Mater. $\mathbf{5}, 065002$ (2010)

67. S. Hornig, T. Heinze, C.R. Becer, U.S. Schubert, Synthetic polymeric nanoparticles by nanoprecipitation. J. Mater. Chem. 19, 3838-3840 (2009) 
68. A. Akbarzadeh, M. Samiei, S. Davaran, Magnetic nanoparticles: preparation, physical properties, and applications in biomedicine. Nanoscale Res. Lett. 7, 144 (2012)

69. V.V. Mody, R. Siwale, A. Singh, H.R. Mody, Introduction to metallic nanoparticles. J. Pharm. Bioallied Sci. 2, 282-289 (2010)

70. T. Ahn, J.H. Kim, H.-M. Yang, J.W. Lee, J.-D. Kim, Formation pathways of magnetite nanoparticles by coprecipitation method. J. Phys. Chem. C 116, 6069-6076 (2012)

71. M.C. Mascolo, Y. Pei, T.A. Ring, Room temperature co-precipitation synthesis of magnetite nanoparticles in a large $\mathrm{pH}$ window with different bases. Materials (Basel, Switzerland) 6, 5549-5567 (2013)

72. M. Unni et al., Thermal decomposition synthesis of iron oxide nanoparticles with diminished magnetic dead layer by controlled addition of oxygen. ACS Nano 11, 2284-2303 (2017)

73. R. Hufschmid et al., Synthesis of phase-pure and monodisperse iron oxide nanoparticles by thermal decomposition. Nanoscale $\mathbf{7}$, 11142-11154 (2015)

74. S. Takami et al., Hydrothermal synthesis of surface-modified iron oxide nanoparticles. Mater. Lett. 61, 4769-4772 (2007)

75. H. Cai et al., Facile hydrothermal synthesis and surface functionalization of polyethyleneimine-coated iron oxide nanoparticles for biomedical applications. ACS Appl. Mater. Interfaces. 5, 1722-1731 (2013)

76. A.B. Chin, I.I. Yaacob, Synthesis and characterization of magnetic iron oxide nanoparticles via w/o microemulsion and Massart's procedure. J. Mater. Process. Technol. 191, 235-237 (2007)

77. S. Santra et al., Synthesis and characterization of silica-coated iron oxide nanoparticles in microemulsion: the effect of nonionic surfactants. Langmuir 17, 2900-2906 (2001)

78. K.V.P.M. Shafi et al., Sonochemical synthesis of functionalized amorphous iron oxide nanoparticles. Langmuir 17, 5093-5097 (2001)

79. A. Hassanjani-Roshan, M.R. Vaezi, A. Shokuhfar, Z. Rajabali, Synthesis of iron oxide nanoparticles via sonochemical method and their characterization. Particuology 9, 95-99 (2011)

80. C. Pascal, J.L. Pascal, F. Favier, M.L. Elidrissi Moubtassim, C. Payen, Electrochemical synthesis for the control of $\gamma-\mathrm{Fe}_{2} \mathrm{O}_{3}$ nanoparticle size. morphology, microstructure, and magnetic behavior. Chem. Mater. 11, 141-147 (1999)

81. F. Dumitrache et al., Highly magnetic $\mathrm{Fe}_{2} \mathrm{O}_{3}$ nanoparticles synthesized by laser pyrolysis used for biological and heat transfer applications. Appl. Surf. Sci. 336, 297-303 (2015)

82. A.A. Bharde et al., Bacteria-mediated precursor-dependent biosynthesis of superparamagnetic iron oxide and iron sulfide nanoparticles. Langmuir 24, 5787-5794 (2008)

83. M.V. Yigit, D. Mazumdar, Y. Lu, MRI detection of thrombin with aptamer functionalized superparamagnetic iron oxide nanoparticles. Bioconjug. Chem. 19, 412-417 (2008)

84. C. Sun et al., In vivo MRI detection of gliomas by chlorotoxin-conjugated superparamagnetic nanoprobes. Small 4, 372-379 (2008)

85. C.G. Hadjipanayis et al., EGFRvIII antibody-conjugated iron oxide nanoparticles for magnetic resonance imaging-guided convectionenhanced delivery and targeted therapy of glioblastoma. Can. Res. 70 6303-6312 (2010)

86. M. Shevtsov et al., Targeting experimental orthotopic glioblastoma with chitosan-based superparamagnetic iron oxide nanoparticles (CS-DXSPIONs). Int. J. Nanomed. 13, 1471-1482 (2018)

87. H.E. Ten Daldrup-Link, Things you might not know about iron oxide nanoparticles. Radiology 284, 616-629 (2017)

88. L.O. Sillerud et al., SPION-enhanced magnetic resonance imaging of Alzheimer's disease plaques in AßPP/PS-1 transgenic mouse brain. $J$ Alzheimer's Dis. 34, 349-365 (2013)

89. T. Fernández, A. Martínez-Serrano, L. Cussó, M. Desco, M. Ramos-Gómez, Functionalization and characterization of magnetic nanoparticles for the detection of ferritin accumulation in Alzheimer's disease. ACS Chem. Neurosci. 9, 912-924 (2018)

90. Z. Zhou et al., Iron/iron oxide core/shell nanoparticles for magnetic targeting MRI and near-infrared photothermal therapy. Biomaterials $\mathbf{3 5}$, 7470-7478 (2014)

91. A. Sood, V. Arora, J. Shah, R.K. Kotnala, T.K. Jain, Multifunctional gold coated iron oxide core-shell nanoparticles stabilized using thiolated sodium alginate for biomedical applications. Mater. Sci. Eng. C 80 , 274-281 (2017)
92. T.R. Pisanic, J.D. Blackwell, V.I. Shubayev, R.R. Fiñones, S. Jin, Nanotoxicity of iron oxide nanoparticle internalization in growing neurons. Biomaterials 28, 2572-2581 (2007)

93. M.V. Yigit, A. Moore, Z. Medarova, Magnetic nanoparticles for cancer diagnosis and therapy. Pharm. Res. 29, 1180-1188 (2012)

94. X. Ji et al., Size control of gold nanocrystals in citrate reduction: the third role of citrate. J. Am. Chem. Soc. 129, 13939-13948 (2007)

95. J.D.S. Newman, G.J. Blanchard, Formation of gold nanoparticles using amine reducing agents. Langmuir 22, 5882-5887 (2006)

96. M.K. Corbierre, R.B. Lennox, Preparation of thiol-capped gold nanoparticles by chemical reduction of soluble $\mathrm{Au}(\mathrm{l})$ - thiolates. Chem. Mater. 17, 5691-5696 (2005)

97. S. Eustis, H.-Y. Hsu, M.A. El-Sayed, Gold nanoparticle formation from photochemical reduction of $\mathrm{Au}^{3+}$ by continuous excitation in colloidal solutions. A proposed molecular mechanism. J. Phys. Chem. B 109, 4811-4815 (2005)

98. M.L. Marin, K.L. McGilvray, J.C. Scaiano, Photochemical strategies for the synthesis of gold nanoparticles from $A u(I I I)$ and $A u(I)$ using photoinduced free radical generation. J. Am. Chem. Soc. 130, 16572-16584 (2008)

99. N.S.M. Yusof, M. Ashokkumar, Sonochemical synthesis of gold nanoparticles by using high intensity focused ultrasound. ChemPhysChem 16, 775-781 (2015)

100. F. Correard et al., Gold nanoparticles prepared by laser ablation in aqueous biocompatible solutions: assessment of safety and biological identity for nanomedicine applications. Int. J. Nanomed. 9, 5415-5430 (2014)

101. Q. Yao et al., Understanding seed-mediated growth of gold nanoclusters at molecular level. Nat. Commun. 8, 927 (2017)

102. V. Sáez, J.T. Mason, Sonoelectrochemical synthesis of nanoparticles. Molecules 14, 4284 (2009)

103. P.K. Jain, K.S. Lee, I.H. El-Sayed, M.A. El-Sayed, Calculated absorption and scattering properties of gold nanoparticles of different size, shape, and composition: applications in biological imaging and biomedicine. J. Phys. Chem. B 110, 7238-7248 (2006)

104. C.L. Zavaleta et al., Multiplexed imaging of surface enhanced Raman scattering nanotags in living mice using noninvasive Raman spectroscopy. Proc. Natl. Acad. Sci. U.S.A. 106, 13511-13516 (2009)

105. H. Hirschberg, S.J. Madsen, Cell mediated photothermal therapy of brain tumors. J. Neuroimmune Pharmacol. 12,99-106 (2017)

106. S. Laurent et al., Magnetic iron oxide nanoparticles: synthesis, stabilization, vectorization, physicochemical characterizations, and biological applications. Chem. Rev. 108, 2064-2110 (2008)

107. W.M. Pardridge, The blood-brain barrier: bottleneck in brain drug development. NeuroRx 2, 3-14 (2005)

108. E.S. Lee, Y.S. Youn, Albumin-based potential drugs: focus on half-life extension and nanoparticle preparation. J. Pharm. Invest. 46, 305-315 (2016)

109. N.M. Smith et al., An unexpected transient breakdown of the blood brain barrier triggers passage of large intravenously administered nanoparticles. Sci. Rep. 6, 22595 (2016)

110. F.-F. An, X.-H. Zhang, Strategies for preparing albumin-based nanoparticles for multifunctional bioimaging and drug delivery. Theranostics $\mathbf{7}$, 3667-3689 (2017)

111. K. Ulbrich, T. Hekmatara, E. Herbert, J. Kreuter, Transferrin- and transferrin-receptor-antibody-modified nanoparticles enable drug delivery across the blood-brain barrier (BBB). Eur. J. Pharm. Biopharm. 71, 251-256 (2009)

112. K. Ulbrich, T. Knobloch, J. Kreuter, Targeting the insulin receptor: nanoparticles for drug delivery across the blood-brain barrier (BBB). J. Drug Target. 19, 125-132 (2011)

113. S.G. Patching, Glucose transporters at the blood-brain barrier: function, regulation and gateways for drug delivery. Mol. Neurobiol. 54, 1046-1077 (2017)

114. R. Gromnicova et al., Glucose-coated gold nanoparticles transfer across human brain endothelium and enter astrocytes in vitro. PLOS ONE $\mathbf{8}$, e81043 (2013)

115. X. Jiang et al., Nanoparticles of 2-deoxy-D-glucose functionalized poly(ethylene glycol)-co-poly(trimethylene carbonate) for dual-targeted drug delivery in glioma treatment. Biomaterials 35, 518-529 (2014) 
116. L. Ji et al., A novel functional CT contrast agent for molecular imaging of cancer. Phys. Med. Biol. 55, 4389 (2010)

117. G. Feng, B. Kong, J. Xing, J. Chen, Enhancing multimodality functional and molecular imaging using glucose-coated gold nanoparticles. Clin. Radiol. 69, 1105-1111 (2014)

118. J.E.G. Barnett, G.D. Holman, K.A. Munday, Structural requirements for binding to the sugar-transport system of the human erythrocyte. Biochem. J. 131, 211-221 (1973)

119. Y. Anraku et al., Glycaemic control boosts glucosylated nanocarrier crossing the BBB into the brain. Nat. Commun. 8, 1001 (2017)

120. G. Xiao, L.-S. Gan, Receptor-mediated endocytosis and brain delivery of therapeutic biologics. Int. J. Cell Biol. 2013, 703545 (2013)

121. C. Saraiva et al., Nanoparticle-mediated brain drug delivery: overcoming blood-brain barrier to treat neurodegenerative diseases. J. Control. Release 235, 34-47 (2016)

122. F.C. Lam et al., Enhanced efficacy of combined temozolomide and bromodomain inhibitor therapy for gliomas using targeted nanoparticles. Nat. Commun. 9, 1991 (2018)

123. D.T. Wiley, P. Webster, A. Gale, M.E. Davis, Transcytosis and brain uptake of transferrin-containing nanoparticles by tuning avidity to transferrin receptor. Proc. Natl. Acad. Sci. U.S.A. 110, 8662-8667 (2013)

124. K.B. Johnsen et al., Targeting transferrin receptors at the blood-brain barrier improves the uptake of immunoliposomes and subsequent cargo transport into the brain parenchyma. Sci. Rep. 7, 10396 (2017)

125. R.J. Boado, Y. Zhang, Y. Zhang, Y. Wang, W.M. Pardridge, GDNF fusion protein for targeted-drug delivery across the human blood-brain barrier. Biotechnol. Bioeng. 100, 387-396 (2007)

126. P. Kumari, B. Ghosh, S. Biswas, Nanocarriers for cancer-targeted drug delivery. J. Drug Target. 24, 179-191 (2016)

127. S. Senapati, A.K. Mahanta, S. Kumar, P. Maiti, Controlled drug delivery vehicles for cancer treatment and their performance. Signal Transd. Targeted Ther. 3, 7 (2018)

128. T.M. Fahmy, P.M. Fong, A. Goyal, W.M. Saltzman, Targeted for drug delivery. Mater. Today 8, 18-26 (2005)

129. Y.H. Bae, K. Park, Targeted drug delivery to tumors: myths, reality and possibility. J. Control. Release 153, 198-205 (2011)

130. H. Gao et al., RGD and interleukin-13 peptide functionalized nanoparticles for enhanced glioblastoma cells and neovasculature dual targeting delivery and elevated tumor penetration. Mol. Pharm. 11, 1042-1052 (2014)

131. S. Wang, Y. Meng, C. Li, M. Qian, R. Huang, Receptor-mediated drug delivery systems targeting to glioma. Nanomaterials 6, 3 (2016)

132. M. Kawamoto, T. Horibe, M. Kohno, K. Kawakami, A novel transferrin receptor-targeted hybrid peptide disintegrates cancer cell membrane to induce rapid killing of cancer cells. BMC Cancer 11, 359 (2011)

133. B. Zhang et al., LDLR-mediated peptide-22-conjugated nanoparticles for dual-targeting therapy of brain glioma. Biomaterials 34, 9171-9182 (2013)

134. H. Xie et al., Lactoferrin-conjugated superparamagnetic iron oxide nanoparticles as a specific MRI contrast agent for detection of brain glioma in vivo. Biomaterials 32, 495-502 (2011)

135. N. Chen et al., Folic acid-conjugated MnO nanoparticles as a $\mathrm{T} 1$ contrast agent for magnetic resonance imaging of tiny brain gliomas. ACS Appl. Mater. Interfaces. 6, 19850-19857 (2014)

136. S.L. Hayward, C.L. Wilson, S. Kidambi, Hyaluronic acid-conjugated liposome nanoparticles for targeted delivery to CD44 overexpressing glioblastoma cells. Oncotarget 7, 34158-34171 (2016)

137. A. Soeda et al., The evidence of glioblastoma heterogeneity. Sci. Rep. $\mathbf{5}$, $7979(2015)$

138. N. Gao, H. Sun, K. Dong, J. Ren, Gold-nanoparticle-based multifunctional amyloid- $\beta$ inhibitor against Alzheimer's disease. Chem. Eur. J. 21, 829-835 (2014)
139. J. Pansieri et al., Multimodal imaging Gd-nanoparticles functionalized with Pittsburgh compound B or a nanobody for amyloid plaques targeting. Nanomed. Nanotechnol. Biol. Med. 12, 1675-1687 (2017)

140. M. Ramakrishnan et al., Selective contrast enhancement of individual Alzheimer's disease amyloid plaques using a polyamine and Gd-DOTA conjugated antibody fragment against fibrillar $A \beta 42$ for magnetic resonance molecular imaging. Pharm. Res. 25, 1861-1872 (2008)

141. M. Plissonneau et al., Gd-nanoparticles functionalization with specific peptides for ß-amyloid plaques targeting. J. Nanobiotechnol. 14,60 (2016)

142. C. Zhang et al., Dual-functional nanoparticles targeting amyloid plaques in the brains of Alzheimer's disease mice. Biomaterials $\mathbf{3 5}$, 456-465 (2014)

143. D. Besong-Agbo et al., Naturally occurring a-synuclein autoantibody levels are lower in patients with Parkinson disease. Neurology $\mathbf{8 0}$, 169-175 (2013)

144. R. Baron, M. Zayats, I. Willner, Dopamine-, I-DOPA-, adrenaline-, and noradrenaline-induced growth of Au nanoparticles: assays for the detection of neurotransmitters and of tyrosinase activity. Anal. Chem. 77, 1566-1571 (2005)

145. Y. Xu, X. Hun, F. Liu, X. Wen, X. Luo, Aptamer biosensor for dopamine based on a gold electrode modified with carbon nanoparticles and thionine labeled gold nanoparticles as probe. Microchim. Acta 182 1797-1802 (2015)

146. R. Huang et al., Gene therapy using lactoferrin-modified nanoparticles in a rotenone-induced chronic Parkinson model. J. Neurol. Sci. 290, 123-130 (2010)

147. F. Zhang, Y.-A. Lin, S. Kannan, R.M. Kannan, Targeting specific cells in the brain with nanomedicines for CNS therapies. J. Controlled Release 240, 212-226 (2016)

148. L. You et al., Targeted brain delivery of rabies virus glycoprotein 29-modified deferoxamine-loaded nanoparticles reverses functional deficits in parkinsonian mice. ACS Nano 12,4123-4139 (2018)

149. A.M. Donson, S.O. Addo-Yobo, M.H. Handler, L. Gore, N.K. Foreman, MGMT promoter methylation correlates with survival benefit and sensitivity to temozolomide in pediatric glioblastoma. Pediatr. Blood Cancer 48, 403-407 (2007)

150. O. Martinho et al., Expression, mutation and copy number analysis of platelet-derived growth factor receptor A (PDGFRA) and its ligand PDGFA in gliomas. Br. J. Cancer 101, 973-982 (2009)

151. J.I.L. Bastien, K.A. McNeill, H.A. Fine, Molecular characterizations of glioblastoma, targeted therapy, and clinical results to date. Cancer 121, 502-516 (2014)

152. J. Cai et al., Detection of ATRX and IDH1-R132H immunohistochemistry in the progression of 211 paired gliomas. Oncotarget 7, 16384-16395 (2016)

153. K.J. Hamblett et al., AMG 595, an anti-EGFRvIll antibody-drug conjugate, induces potent antitumor activity against EGFRvIll-expressing glioblastoma. Mol. Cancer Ther. 14, 1614-1624 (2015)

154. H. Tong, K. Lou, W. Wang, Near-infrared fluorescent probes for imaging of amyloid plaques in Alzheimer's disease. Acta Pharmaceutica Sinica B 5, 25-33 (2015)

155. M.S. Gunay, A.Y. Ozer, S. Chalon, Drug delivery systems for imaging and therapy of Parkinson's disease. Curr. Neuropharmacol. 14, 376-391 (2016)

156. T.T. Sibov et al., Umbilical cord mesenchymal stem cells labeled with multimodal iron oxide nanoparticles with fluorescent and magnetic properties: application for in vivo cell tracking. Int. J. Nanomed. $\mathbf{9}$, 337-350 (2014)

157. K. Hu et al., Neuroprotective effect of gold nanoparticles composites in Parkinson's disease model. Nanomed. Nanotechnol. Biol. Med. 14, 1123-1136 (2018) 